\title{
A Histological Study on the Possible Adverse Effect of Sofosbuvir on Lungs of Adult Male Albino Rats
}

\author{
Original \\ Sara Adel Hosny, Manal Ali Abdel Mohsen, Marwa Mohamed Sabry \\ Article \\ Departments of of Histology, Faculty of Medicine, Cairo university, Egypt.
}

\begin{abstract}
Background: In 2011, three new direct-acting antivirals (DAAs) for HCV including sofosbuvir were accredited for HCV management.

Aim: To evaluate the impact of Sofosbuvir treatment on the histological structure of the lungs of adult male albino rat.

Materials and Methods: 15 male rats were classified into: control group of 5 rats and sofosbuvir treated group of 10 rats that received sofosbuvir orally in a dose of $4 \mathrm{mg} / \mathrm{kg}$ per day for 5 weeks. Blood samples were drawn to assess serum Transforming growth factor- $\beta$ (TGF- $\beta$ ). Lung specimens were obtained for evaluation by light microscope. Alpha smooth Muscle Actin $(\alpha$-SMA), E-cadherin immunohistochemistry, and statistical analysis were applied.

Results: Marked increase in the mean value of serum TGF- $\beta$ in Sofosbuvir treated group when compared to control group. Sofosbuvir resulted in histological and immunohistochemical changes in the lungs of sofosbuvir treated rats.

Conclusion: Severe lung injury was detected after treatment with sofosbuvir. However further studies should be done to determine the exact mechanism and more caution should be taken during administration of sofosbuvir.
\end{abstract}

Key Words: Alpha smooth muscle actin, E-cadherin, Lung, sofosbuvir, TGF- $\beta$.

Revised: 23 June 2020, Accepted: 10 July 2020.

Corresponding Author: Sara Adel Hosny, MD, Department of Histology, Faculty of Medicine, Cairo University, Egypt, Tel.: 01005625595, E-mail: dr.sara_adelh@hotmail.com

ISSN:2536-9172, June 2020, Vol. 4, No. 1

\section{INTRODUCTION}

Infection of the liver with hepatitis $\mathrm{C}$ virus (HCV) causes inflammation of the liver called Hepatitis C, which can be either acute or chronic. In acute hepatitis $\mathrm{C}$, symptoms appear quickly and persist for few weeks. However, symptoms in chronic hepatitis $\mathrm{C}$ is established over a period of months and may not be obvious early. In contrast to hepatitis $\mathrm{A}$ and $\mathrm{B}$, hepatitis $\mathrm{C}$ has no vaccine, although several attempts was done to create one. Hepatitis $\mathrm{C}$ is highly infectious; the World Health Organization (WHO) estimates that 170 million are infected ${ }^{[1,2]}$.

In treatment of chronic HCV infection, the principal target is to accomplish a sustained virologic response (SVR), clarified as undetectable HCV-RNA, 24weeks after finishing the treatment program ${ }^{[3,4]}$. Until 2011, the authorized treatment for chronic HCV infection for 48 weeks was in the form of combination of ribavirin and pegylated interferon (Peg IFN). After treatment, genotype 1 (G1) SVR was $40 \%$ to $50 \%$ and genotypes 2 and 3 (G2/G3) SVR were $70 \%$ to $80 \%{ }^{[5,6]}$.

The new three direct-acting antivirals (DAAs) for HCV were authorized in 2011 for utilization as a part of $\mathrm{HCV}$ combination therapies. They consists of daclatasvir, simeprevir and sofosbuvir. When DAAs usage have been approved, IFN-free combinations were occasionally utilized. HCV-RNA polymerase inhibitors as Sofosbuvir exhibit significant difficulty for establishment of resistance. It is a great benefit as other drugs of HCV that attack protease develop rapid resistance leading to therapeutic failure ${ }^{[7,8]}$.

However, it was revealed that Sofosbuvir treatment manifests prevalent side effects as memory impairment, gastrointestinal reflux, dyspnea, chest pain, muscle spasm, blurred vision, depression, migraine and alopecia ${ }^{[9]}$.

Accordingly, this research was designed to evaluate the impact of Sofosbuvir treatment on the histological structure of the lungs of adult male albino rat.

\section{MATERIALS AND METHODS}

\section{Animals:}

This research included 15 male adult albino rats with a mean body weight ranging between 170 and 200 grams. They were provided by Kasr Al-Ainy animal house, Faculty of Medicine, Cairo University. They were sheltered in sanitary cages in agreement with animal research principles provided by the National Institute of Health and authorized by Animal Ethics Committee. The rats received chow and water ad libitum. 


\section{Rats were categorized into the subsequent groups:}

1. Control group (Group A): It comprised five rats that received distilled water orally using gastric tube daily for 5 weeks.

2. Sofosbuvir treated group (Group B): comprised ten rats, which received Sofosbuvir (MPI Viropack, 400mg, Sofosbuvir, Marcyrl Pharmaceuticals). Distilled water was used for dissolving the tablets to be given to the rats orally using gastric tube for 5 weeks in a dose of $4 \mathrm{mg} /$ $\mathrm{kg}$ per day ${ }^{[10]}$.

After 5 weeks, ketamine hydrochloride $(50 \mathrm{mg} / \mathrm{kg}$; Sigma) intramuscular injection was used to anaesthetize the rats ${ }^{[11]}$. Blood samples were collected to measure the serum pro-fibrotic marker, transforming growth factor$\beta$ (TGF- $\beta$ ) in the Biochemistry Department, Faculty of Medicine, Cairo University.

Lung specimens from groups [A and B] were dissected out and subjected to the following:

Part (I): Specimens from left lung were fixed in $10 \%$ formol saline solution to be processed into paraffin blocks. Serial sections at 7 um thicknesses were cut using a microtome and mounted on glass slides. Other sections were mounted on positive charged slides for immunohistochemistry.

Part (II): Specimens from right lung were fixed in $2.5 \%$ glutaraldehyde to be processed for preparation of semithin sections.

\section{- Histological study:}

\section{1- Light microscopic study:}

(A) Paraffin sections were subjected to the following stains:

1-Haematoxylin and $\operatorname{Eosin}^{[12]}$.

2- Masson's Trichrome staining ${ }^{[13]}$.

3-Immunohistochemical staining ${ }^{[13]}$.

For immunohistochemistry, lung sections were mounted on positive charged slides, deparaffinized and rehydrated. Tissue sections were boiled in $10 \mathrm{mM}$ citrate buffer for Antigen retrieval. The sections were incubated in hydrogen peroxide to inhibit endogenous peroxidase activity. Sections were incubated overnight with:

a) Rabbit monoclonal anti- $\alpha$ SMA [E184] (ab32575, Abcam plc, England).

b) Mouse monoclonal anti E-cadherin antibody (ab1416, Abcam plc, England).
The labeled avidin-biotin-peroxidase complex (Histostain SP kit, Zymed Laboratories Inc, San Francisco, USA) was used for immune detection of the bound primary antibody. Immunohistochemical-staining specificity was created by using Diaminobenzidine (DAB) as a chromogen and Meyer's haematoxylin as a counterstain. Processing of negative control sequential sections were done by replacement of the primary antibody by phosphate buffer saline with performing all other stages in the same technique. Positive tissue control for alpha smooth muscle actin ( $\alpha$-SMA) immunostaining was human uterus with a brownish cytoplasmic immunoreaction while that of E-cadherin was intercellular junction marker in normal human skin tissue provided by the manufacture (abcam).

(B) Semithin sections ${ }^{[12]}$ :

Small pieces of right lung specimen with average size of $1 \mathrm{~mm}^{3}$ were immediately cut and rapidly fixed in $2.5 \%$ phosphate buffered glutaraldehyde (PH 7.2), post fixed in $1 \%$ osmium tetroxide and embedded in resin. Semithin sections $(2 \mu \mathrm{m})$ were cut and stained with toluidine blue for 45 seconds to be examined with the light microscope.

\section{2- Morphometric study:}

Data were acquired using "Leica Qwin 500 C" image analyzer computer system in Histology department, Faculty of Medicine, Cairo University. The mean number of pneumocytes (type II), mean area percent of collagen fibers, mean area percent of smooth muscle actin and E-cadherin immunoreactivity were assessed in 10 nonoverlapping fields for each slide of all specimens at a magnification of 400 .

\section{3- Statistical Analysis:}

SPSS software version 9 (SPSS, Chicago, IL) was used to evaluate the acquired measurements. Comparing between different groups was formulated by analysis of variance (ANOVA) followed by post hoc Tukey test. Outcomes were articulated in the form of means \pm standard deviation (SD). The variances were regarded statistically significant when "p value" was $<0.05$ (14).

\section{RESULTS}

\section{Biochemical results (Histogram 1):}

The mean value of serum TGF- $\beta$ in control group was (5.868 \pm 0.38$)$. In Sofosbuvir treated group was (9.42 \pm 0.29$)$, which was significantly increased $(P<0.05)$ in comparison with control group. 
Histogram 1: Mean serum level of TGF- $\beta$ in the studied groups

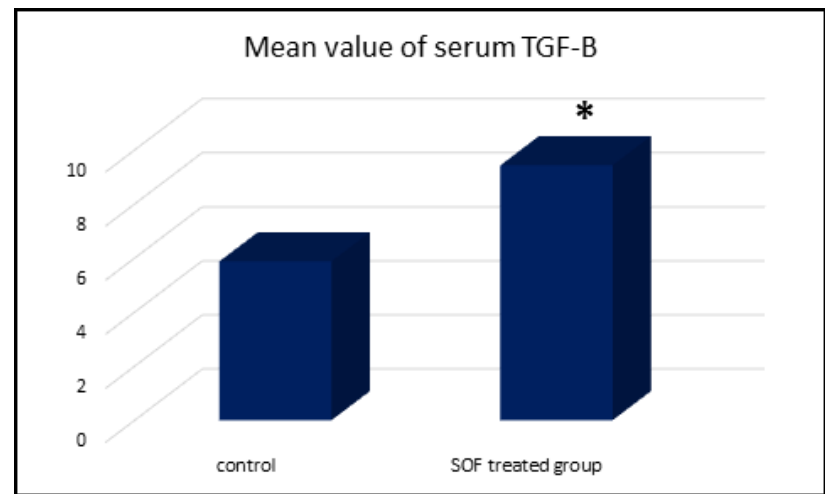

*Significant as compared to control group (A) $(p<0.05)$.

\section{Histological Results:}

\section{Haematoxylin and Eosin Stained Sections (Plate. 1):}

Control group exhibited normal spongy lung pattern in the form of bronchioles lined with cuboidal cells associated with club cells, alveolar duct formed of lung alveoli with muscle knobs. Lung alveoli are patent, thin walled saclike structures lined with numerous flat elongated cells (pneumocytes type I) with few cuboidal cells (pneumocytes type II). Sofosbuvir (SOF) treated group exhibited a small bronchiole showing desquamated cells and acidophilic material in its lumen. Focal parts of the lung tissue showed the interalveolar septa were thickened, infiltrated by mononuclear cells causing alveolar narrowing (shrunken alveoli) associated with vascular congestion.

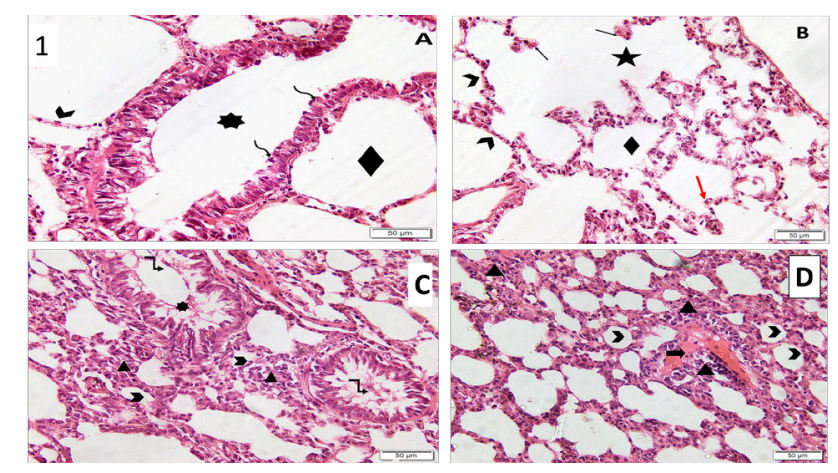

Plate (1): Photomicrographs of lung sections in rats of:

- $\quad$ A\&B (control group): Showing bronchioles lined with cuboidal cells (asterisks) associated with club cells (curved arrows), alveolar ducts (star) formed of lung alveoli with muscle knobs (arrows). Lung alveoli are patent, thin walled sac-like structures (rhombus) lined with flat elongated cells (pneumocytes type I) (arrow heads) with few cuboidal cells (pneumocytes type II) (red arrow).

- C\&D (Sofosbuvir treated group): showing a small bronchiole showing desquamated cells (kinked arrows) and acidophilic material in its lumen (Asterisks). The interalveolar septa are thickened, infiltrated by mononuclear cells (pyramids) causing alveolar narrowing (arrowhead) associated with vascular congestion (thick arrow).

(H\&E x200).
Semithin Sections stained with toluidine blue (Plate. 2)::

Control group exhibited thin walled lung alveoli lined with flat elongated cells (pneumocytes type I) having flat nuclei and few cuboidal cells (pneumocytes type II) having central pale vesicular nucleus and foamy cytoplasm. Sofosbuvir (SOF) treated group showed thickening of the alveolar wall and shrunken lumen. The interalveolar septa is thickened, showing mononuclear cells, mainly macrophages with eccentric large vesicular indented nuclei. Alveolar wall shows apparent decreased pneumocytes type I with many type II pneumocytes. Many spindle shaped fibroblast-like cells with flat nuclei can be seen.

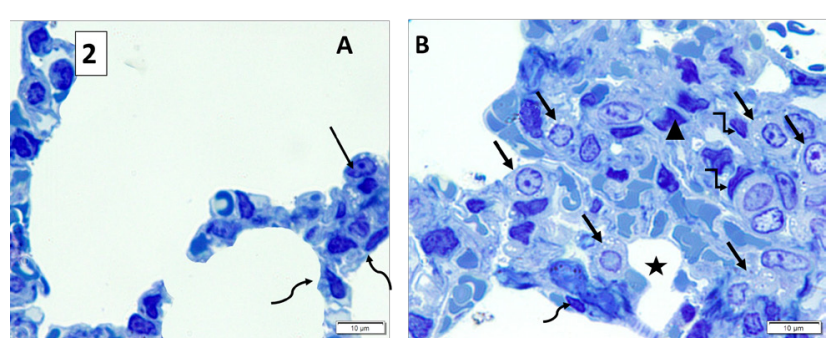

Plate (2): Photomicrographs of semithin sections in rat lungs stained with toluidine blue of:

- A (control group): Thin walled lung alveoli lined with flat elongated cells (pneumocytes type I) having flat nuclei (Curved arrows) with few cuboidal pneumocytes type II having central pale vesicular nucleus (arrow).

- B (Sofosbuvir treated group): exhibiting thickening of the interalveolar septa with shrunken alveolar lumen (stars). The interalveolar septum shows mononuclear cells, mainly macrophages with eccentric large vesicular indented nuclei (triangles). Alveolar wall shows apparent decreased pneumocytes type I (curved arrow) with many pneumocytes type II (arrows). Many spindle shaped fibroblast-like cells (kinked arrow) with flat nuclei can be seen.

(Semithin sections $\mathrm{x} 1000)$.

\section{Masson Trichrome Stained Sections (Plate. 3):}

Examination of lung sections revealed minimal collagen fibers in control group. On the other hand lung sections in Sofosbuvir (SOF) treated group revealed dense collagen fiber deposition in interalveolar septa.

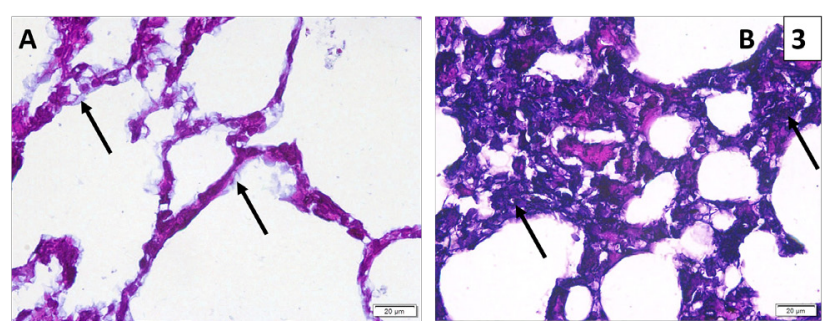

Plate (3): Photomicrographs of sections stained with Masson's trichrome in rat lungs of:

- A (control group): exhibiting minimal collagen fibers in Interalveolar septa (arrows).

- B (Sofosbuvir treated group): exhibiting dense collagen fiber deposition (arrows) in thickened interalveolar septa.

(Masson's trichrome x400). 
Immunohistochemically stained sections for alpha Smooth muscle actin (Plate. 4):

Sections in the control rat lung showed minimal immunostaining with $\alpha$-smooth muscle actin mostly within the muscle knobs of the alveolar duct wall. While, Sofosbuvir (SOF) treated group showed increased immunoreactivity of $\alpha$-SMA in the cytoplasm of myofibroblasts in interalveolar septum as compared to the control group.

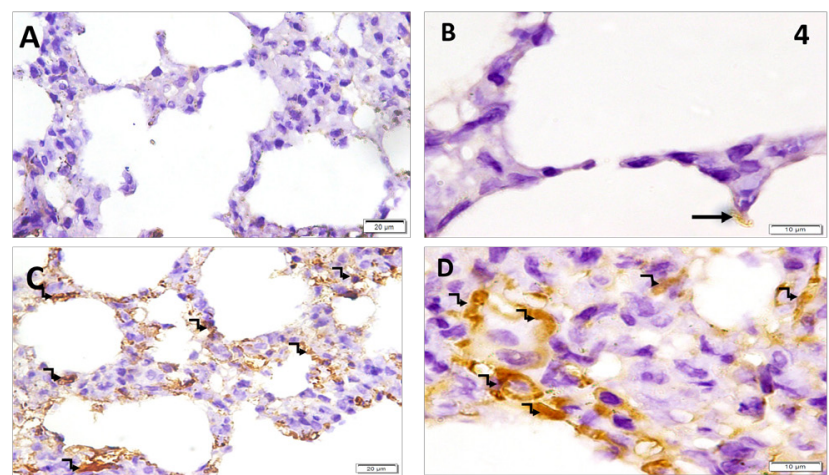

Plate (4): Photomicrographs of immunohistochemical stained sections with anti- $\alpha$ Masson antibody in rat lungs:

- $\quad$ A\&B (control group): showing faint cytoplasmic immunostaining with $\alpha$-smooth muscle actin mostly within muscle knobs in the alveolar duct wall (arrow).

- C\&D (Sofosbuvir treated group): showing obvious immunostaining with $\alpha$-smooth muscle actin in the cytoplasm of myofibroblasts (Kinked arrows) in interalveolar septum.

( $\alpha$-SMA immunostaining: A\&C x400; B, D x1000).

Immunohistochemically stained sections for E-cadherin(Plate. 5):

Examination of lung sections in control group showed intercellular immunostaining with E-cadherin between cells lining bronchioles and alveolar cells. While, Sofosbuvir (SOF) treated group showed minimal intercellular immunostaining with E-cadherin between alveolar cells when compared to the control group.

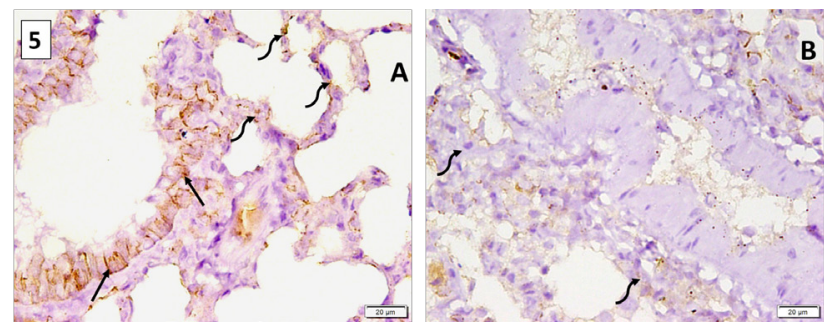

Plate (5): Photomicrographs of immunohistochemical stained sections with anti-E cadherin antibody in rat lungs:

- A (control group): revealing marked and widely distributed membranous immunostaining of the intercellular E-cadherin in cells lining bronchioles (arrows) and alveolar cells (curved arrows).

B (Sofosbuvir treated group): showing minimal intercellular immunostaining with E-cadherin between cells alveolar cells (arrows). $\quad$ (E-cadherin immunostaining x400).

\section{Quantitative Morphometric Results:}

1- Mean number of pneumocytes type II in the investigated groups (Histogram 2):

Mean number of pneumocytes type II in the control group was (2 \pm 0.67$)$. In Sofosbuvir (SOF) treated group was $(9.3 \pm 1.16)$, which was significantly elevated $(P<0.05)$ in comparison with control group.

2- Mean Area Percent of collagen fibers in investigated Groups (Histogram 2):

The mean area percent of collagen fibers stained with Masson's trichrome in control group was (8.71 11.29$)$. In Sofosbuvir (SOF) treated group was $(20.23 \pm 1.17)$, which was significantly elevated $(P<0.05)$ in comparison with control group.

Histogram 2: Mean number of pneumocytes type II and mean area $\%$ of collagen fibers in the investigated groups

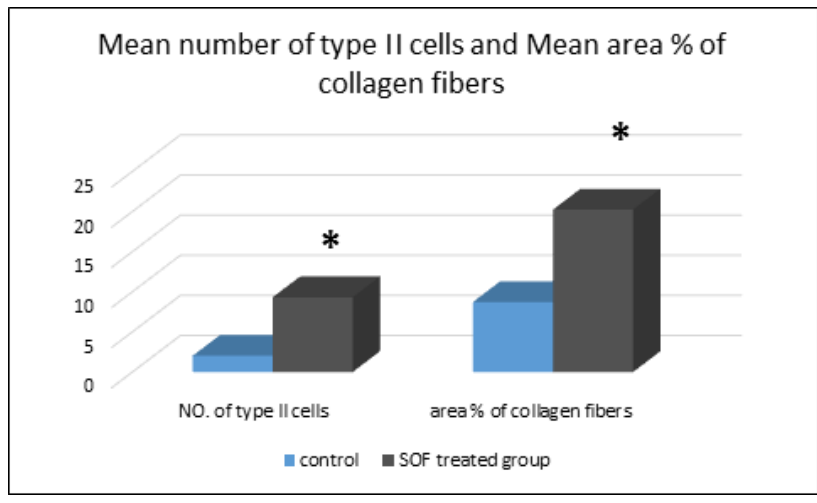

*Significant as compared to control group (A) $(p<0.05)$

3- Mean Area Percent of alpha smooth muscle actin immunoreactivity in the investigated Groups (Histogram 3):

The mean area $\%$ of $\alpha$-SMA immunoreactivity in control group was $(9.109 \pm 4.15)$. In Sofosbuvir (SOF) treated group was $(40.85 \pm 7.94)$, which was significantly elevated $(P<0.05)$ in comparison with control group.

\section{4- Mean Area Percent of E-cadherin immunoreactivity in the investigated Groups (Histogram 3):}

The mean area \% of E-cadherin immunoreactivity in control group was (7.32 \pm 1.09$)$. In Sofosbuvir (SOF) treated group was $(2.48 \pm 0.61)$, which was significantly reduced $(P<0.05)$ in comparison with control group. 
Histogram 3: Mean area \% of $\alpha$-SMA immunoreactivity and E-cadherin immunoreactivity in the investigated groups

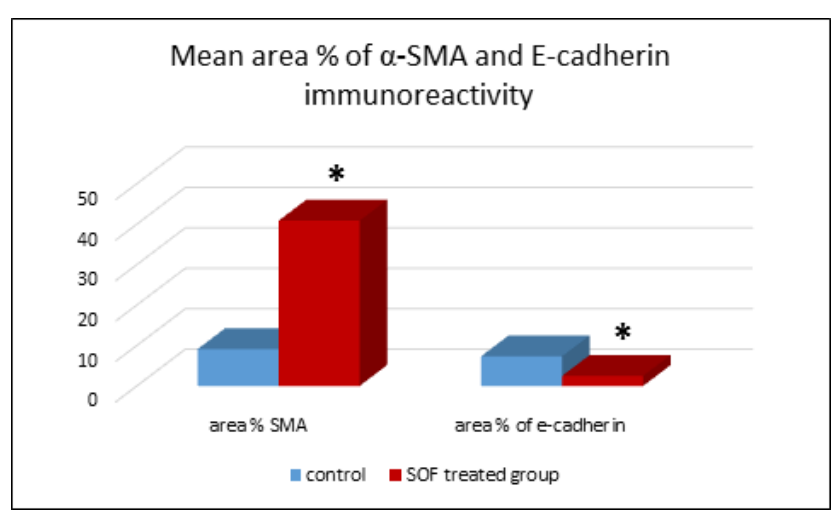

*Significant as compared to control group (A) $(p<0.05)$

\section{DISCUSSION}

Patients infected with HCV are successfully treated with direct-acting antiviral agents (DAAs) that inhibits viral replication. All hepatitis $\mathrm{C}$ virus genotypes are affected by Sofosbuvir, which is a nucleotide analogue polymerase inhibitor ${ }^{[15-17]}$.

The common adverse effects of simeprevir (SIM) and sofosbuvir (SOF) include fatigue, headache and nausea. However, patients in Helmers et al., 2015 ${ }^{[18]}$ study developed severe lung injury with respiratory failure that did not cure with corticosteroids and ended by patients' death.

Hence, the aim of this research was to evaluate the impact of Sofosbuvir treatment on the histological structure of the lungs of adult male albino rat.

In the present study, the mean value of serum TGF- $\beta$ in Sofosbuvir treated group showed marked increase when compared to control group. Haematoxylin and Eosin stained sections and the semithin sections in the same group displayed small bronchiole showing desquamated cells and acidophilic material in its lumen. The interalveolar septa were thickened, infiltrated by mononuclear cells mainly macrophages with eccentric large vesicular indented nuclei causing alveolar narrowing (shrunken alveoli) associated with vascular congestion. Alveolar wall shows apparent decreased pneumocytes type I with many pneumocytes type II. Many spindle shaped fibroblast-like cells with flat nuclei can be seen.

This was in agreement with Helmers et al., 2015 who stated that administration of simeprevir (SIM) and sofosbuvir (SOF) caused lung injury that was detected in surgical lung biopsy revealing fibrosis with scattered eosinophils, increased alveolar macrophages and highly vacuolated pneumocytes type II.
In the current study a statistically significant increase in the number of pneumocytes type II was detected in sofosbuvir treated group, this was in agreement with the study of Helmers et al., 2015. The increase in the number of pneumocytes type II could be explained as a trial to repair by these cells. Pneumocytes type II are considered to be progenitor cells. They are able of self-renewal and differentiation into pneumocytes type I after exposure to injury under the effect of TGF- $\beta$, this could clarify the significant increase of TGF- $\beta$ in sofosbuvir treated group in the present work. ${ }^{[19]}$

Pulmonary fibrosis is a disease characterized by sustained matrix deposition or defective matrix degradation and breakdown of the lung parenchyma, causing respiratory failure and high mortality ${ }^{[20]}$. In Lung injury, the alveolar epithelium (both type I and II pneumocytes) was reported to be damaged with interrupted basement membrane and capillary wall, with increased number of inflammatory cells. Elevation of Inflammatory chemokines and cytokines as tumor necrosis factor- $\alpha$ and interleukin- 1 was reported to be followed by leakage of proteins (pro-fibrotic markers as TGF- $\beta 1$, fibrin and fibronectin) into the alveolar spaces and interalveolar tissue. This induced proliferation and differentiation of fibroblast and myofibroblasts. They deposit matrix causing fibrosis and destruct the lung parenchyma $^{[21,22]}$. In this view, the present study showed significant increase of mean area percent of collagen fibers in Masson's Trichrome stained sections associated with marked elevation in the mean area percent of myofibroblast $\alpha$-SMA immunoreactivity in Sofosbuvir treated group in comparison with control group. This might point to the potentiality of sofosbuvir to affect the number and activity of the myofibroblasts with consequence collagen fibers accumulation. This could be supported by the work of Helmers et al., 2015 $5^{[18]}$ who stated that sofosbuvir induces lung fibrosis. Alpha-smooth muscle actin ( $\alpha$-SMA) a constituent of myofibroblasts, is considered an activated fibroblasts marker. Myofibroblasts differentiation was reported to be induced by TGF- $\beta$ and these cells are responsible for extracellular matrix assembly consisting of collagen and fibronectin. In addition, TGF- $\beta$ stimulates integrins, matrix metalloproteinases and protease inhibitors. In addition, it suppresses the production of antifibrotic agents as prostaglandin $\mathrm{E} 2^{[23-25]}$.

Consistent with TGF- $\beta$ and $\alpha$-SMA immunoreactivity results, the E-cadherin immunoreactivity results showing significant decrease of E-cadherin immunoreactivity in sofosbuvir treated group in comparison with control group. Going with this finding, it was reported that TGF- $\beta$ increased expression of $\alpha$-SMA, vimentin and desmin was associated with suppression of tight junction and adherens junction proteins as claudin, zonula occludens (ZO-1) and E- cadherin ${ }^{[26]}$. Proper tight and adherence junction formation is mandatory for maintenance of epithelial barrier function. Adherens junction includes 
E-cadherin transmembrane protein, is important for apical and basolateral polarization and epithelial cells adhesion; regulating epithelial proliferation and differentiation and suppressing intracellular signaling pathways ${ }^{[27,28]}$. Impairment of E-cadherin expression indicates decreased packing and organization of the cells with the defectiveness of epithelial cell integrity ${ }^{[29]}$. This is in accordance with our findings that showed sloughing of epithelial cells in Sofosbuvir treated group with reduction in E-cadherin expression.

Based upon outcomes of the current study, it could be concluded that after five weeks of sofosbuvir administration, sever lung injury was developed. However, further studies using different doses and durations should be done to determine the exact mechanism and more caution should be taken during administration of sofosbuvir

\section{CONFLICT OF INTEREST}

There are no conflicts of interest.

\section{REFERENCES}

1. Maheshwari A, Ray S, Thuluvath PJ. Acute hepatitis C. Lancet 2008; 372(9635): 321-332.

2. Webster DP, Klenerman P, Dusheiko GM. Hepatitis C. Lancet 2015; 385(9973): 1124-1135.

3. Bruno S, Stroffolini T, Colombo M, Bollani S, Benvegnù L, Mazzella G, Ascione A, Santantonio T, Piccinino F, Andreone P, Mangia A, Gaeta GB, Persico M, Fagiuoli S, Almasio PL, Italian Association of the Study of the Liver Disease (AISF). Sustained virological response to interferon-alpha is associated with improved outcome in HCV-related cirrhosis: a retrospective study. Hepatology 2007; 45(3): 579-587.

4. Van der Meer AJ, Veldt BJ, Feld JJ, Wedemeyer H, Dufour JF, Lammert F, Duarte-Rojo A, Heathcote EJ, Manns MP, Kuske L, Zeuzem S, Hofmann WP, de Knegt RJ, Hansen BE, Janssen HL. Association between sustained virological response and allcause mortality among patients with chronic hepatitis $\mathrm{C}$ and advanced hepatic fibrosis. JAMA: the journal of the American Medical Association 2012; 308(24): 2584-2593.

5. Fried MW, Shiffman ML, Reddy KR, Smith C, Marinos G, Gonçales FL Jr, Häussinger D, Diago M, Carosi G, Dhumeaux D, Craxi A, Lin A, Hoffman J, Yu J. Peginterferon alfa-2a plus ribavirin for chronic hepatitis $\mathrm{C}$ virus infection. The New England journal of medicine 2002; 347(13): 975-982.
6. Jacobson IM, McHutchison JG, Dusheiko G, Di Bisceglie AM, Reddy KR, Bzowej NH, Marcellin P, Muir AJ, Ferenci P, Flisiak R, George J, Rizzetto M, Shouval D, Sola R, Terg RA, Yoshida EM, Adda N, Bengtsson L, Sankoh AJ, Kieffer TL, George S, Kauffman RS, Zeuzem S; ADVANCE Study Team. Telaprevir for previously untreated chronic hepatitis $\mathrm{C}$ virus infection. N Engl J Med 2011; 364: 2405-2416.

7. Eric L, Alessandra M, David W, ZeidKayali L. Sofosbuvir for Previously Untreated Chronic Hepatitis C Infection. N engl j med 2013; 368-320.

8. European Association for the Study of the Liver (EASL). Recommendations on treatment of hepatitis. J. Hepatol.2015; 63: 199-236.

9. Bhatia HK, Singh H, Grewal N, Natt NK. Sofosbuvir: A novel treatment option for chronic hepatitis C infection. Journal of Pharmacology \&Pharmacotherapeutics 2014; 5(4): 278-84.

10. Issa NM, El-Sherif NM. Histological and Immunohistochemical Studies on the Cornea and Retina of Sofosbuvir Treated Rats. Austin J Anat 2017; 4(2): 1068 .

11. Struck MB, Andrutis KA, Ramirez HE, Battles AH. Effect of a short-term fast on ketaminexylazine anesthesia in rat. J Am Assoc Lab Anim Sci 2011; 50(3): 344-348.

12. Kiernan J. Histological and Histochemical Methods: Theory and Practice.5th ed., Scoin publisher. United Kingdom; 2015. 111-162.

13. Suvarna SK, Layton C, Bancroft JD. Bancroft's theory and practice of histological techniques, 7th edition. Elsevier Health sciences, Churchill Livingstone; 2012: 381-426 and 493-538.

14. Emsley R, Dunn G, White IR. Mediation and moderation of treatment effects in randomized controlled trials of complex interventions. Start Methods Med Res.2010; 19(3): 237-270.

15. Murakami E, Tolstykh T, Bao H, Niu C, Steuer HM, Bao D, Chang W, Espiritu C, Bansal S, Lam AM, Otto MJ, Sofia MJ, Furman PA. Mechanism of activation of PSI-7851 and its diastereoisomer PSI-7977. J Biol Chem. 2010; 285: 34337-34347.

16. Chhatwal J, Wang X, Ayer T, Kabiri M, Chung RT, HurC, Donohue JM, Roberts MS, Kanwal F. Hepatitis $\mathrm{C}$ disease burden in the United States in the era of oral direct-acting antivirals. Hepatology 2016; 64: 1442-1450.

17. 17. Asselah T, Boyer N, Saadoun D, MartinotPeignoux M, Marcellin P. Direct-acting antivirals for the treatment of hepatitis $\mathrm{C}$ virus infection: optimizing current IFN-free treatment and future perspectives. Liver Int 2016; 36: Suppl 1:47-57. 
18. Helmers RA, Byrne TJ, Wesselius LJ, Leslie KO. Serious Pulmonary Toxicity Secondary to Novel Hepatitis C Antiviral Therapy in a Liver Transplant Recipient. Mayo Clin Proc. 2015; 90(9): 1294-1297.

19. Barkauskas CE, Cronce MJ, Rackley CR, Bowie EJ, Keene DR, Stripp B.R, Randell SH, Noble PW, Hogan BL. Type 2 alveolar cells are stem cells in adult lung. J. Clin. Investig. 2013; 123, 3025-3036.

20. Wuyts WA, Agostini C, Antoniou KM, Bouros D, Chambers RC, Cottin V, Egan JJ, Lambrecht BN, Lories R, Parfrey H, Prasse A, Robalo-Cordeiro C, Verbeken E, Verschakelen JA, Wells AU, Verleden GM. The pathogenesis of pulmonary fibrosis: a moving target. Eur Respir J 2013; 41: 1207-1218.

21. Hardie WD, Glasser SW, Hagood JS. Emerging concepts in the pathogenesis of lung fibrosis. Am J Pathol 2009; 175: 3-16.

22. Barratt SL, Creamer A, Hayton C, Chaudhuri N. Idiopathic Pulmonary Fibrosis (IPF): An Overview. J. Clin. Med. 2018; 7, 201.

23. Krafts KP. Tissue repair: the hidden drama. Organogenesis2010;6 (4), 225-233.

24. Bauman KA, Wettlaufer SH, Okunishi K, Vannella KM, Stoolman JS, Huang SK, Courey AJ, White ES, Hogaboam CM, Simon RH. The antifibrotic effects of plasminogen activation occur via prostaglandin E2 synthesis in humans and mice. J. Clin. Investig 2010; 120, 1950-1960.
25. Horie M, Saito A, Noguchi S, Yamaguchi Y, Ohshima M, Morishita Y, Suzuki H.I, Kohyama T, Nagase T. Differential knockdown of TGF- $\beta$ ligands in a three-dimensional co-culture tumorstromal interaction model of lung cancer. BMC Cancer 2014; 14, 580.

26. Shirakihara T, Saitoh M, Miyazono K. Differential regulation of epithelial and mesenchymal markers by delta EF1 proteins in epithelial mesenchymal transition induced by TGF-beta. Mol. Biol. Cell 2007; 18, 3533-3544.

27. Tam A, Wadsworth S, Dorscheid D, Man SF, Sin DD. The airway epithelium: more than just a structural barrier. Ther. Adv. Respir. Dis.2011; 5, 255-273.

28. Nawijn MC, Hackett TL, Postma DS, Van Oosterhout AJ, Heijink IH. E-cadherin: gatekeeper of airway mucosa and allergic sensitization. Trends Immunol. 2011; 32, 248-255.

29. Post S, Heijink IH, Hesse L, Koo HK, Shaheen F, Fouadi M, Kuchibhotla VNS, Lambrecht BN, Van Oosterhout AJM, Hackett TL, Nawijn Mc. Characterization of a lung epithelium specific E-cadherin knock-out model: Implications for obstructive lung pathology. Sci Rep. 2018; 8, 13275 . 